\title{
Diversity and evolution of mariner-like elements in aphid genomes
}

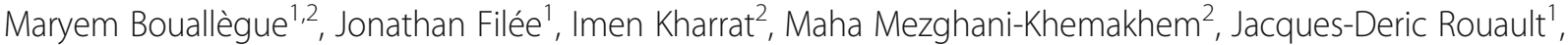 \\ Mohamed Makni ${ }^{2}$ and Pierre Capy ${ }^{1 *}$
}

\begin{abstract}
Background: Although transposons have been identified in almost all organisms, genome-wide information on mariner elements in Aphididae remains unknown. Genomes of Acyrthosiphon pisum, Diuraphis noxia and Myzus persicae belonging to the Macrosiphini tribe, actually available in databases, have been investigated.

Results: A total of 22 lineages were identified. Classification and phylogenetic analysis indicated that they were subdivided into three monophyletic groups, each of them containing at least one putative complete sequence, and several non-autonomous sublineages corresponding to Miniature Inverted-Repeat Transposable Elements (MITE), probably generated by internal deletions. A high proportion of truncated and dead copies was also detected. The three clusters can be defined from their catalytic site: (i) mariner DD34D, including three subgroups of the irritans subfamily (Macrosiphinimar, Batmar-like elements and Dnomar-like elements); (ii) rosa DD41D, found in A. pisum and D. noxia; (iii) a new clade which differs from rosa through long TIRs and thus designated LTIR-like elements. Based on its catalytic domain, this new clade is subdivided into DD40D and DD41D subgroups. Compared to other Tc1/mariner superfamily sequences, rosa DD41D and LTIR DD40-41D seem more related to maT DD37D family.
\end{abstract}

Conclusion: Overall, our results reveal three clades belonging to the irritans subfamily, rosa and new LTIR-like elements. Data on structure and specific distribution of these transposable elements in the Macrosiphini tribe contribute to the understanding of their evolutionary history and to that of their hosts.

Keywords: Aphids, Comparative genomics, Tc1-mariner, Transposable elements, MITEs, Molecular evolution

\section{Background}

Genomes contain diverse repetitive DNA sequences of transposable elements (TEs), contributing to their plasticity, adaptability and evolution [1-3]. Class II TEs use a "cut and paste" mechanism. They are either autonomous transposons encoding their own transposase or nonautonomous transposons including truncated copies (i.e. copies with only one or no extremity) or copies with internal deletions, but with two intact extremities. Although not encoding for a functional transposase, these shorter copies or miniature inverted repeat transposable elements (MITEs) can be trans-mobilized and may reach high copy number with a size homogeneity

\footnotetext{
* Correspondence: pierre.capy@egce.cnrs-gif.fr

${ }^{1}$ Laboratoire Evolution, Génomes, Comportement, Ecologie CNRS, Université Paris-Sud, IRD, Université Paris-Saclay, 1 avenue de la Terrasse, 91198 Gif-sur-Yvette Cedex, France

Full list of author information is available at the end of the article
}

that distinguishes them from other non-autonomous elements [4].

The Tc1/mariner superfamily is ubiquitous and forms the largest group of eukaryotic Class II TEs [5]. Its members share several common characteristics and synapomorphies. In particular, the target insertion site is TA, the ORF of autonomous copies encodes a transposase of 282 to 350 amino-acid residues [6]; the transposase contains two helix-turn-helix (HTH) motifs in DNA binding domains and a catalytic triad DDE/D motif $[5,7]$.

Despite these similarities, two major differences can separate families of Tc1/mariner: (i) their complete length from 1 to $5 \mathrm{~kb}$ due to their TIR (i.e. the marinerlike element $M L E$ 13-34 bp long, the Tc1-like element TLE ranging from 20 to $600 \mathrm{bp}$ ), (ii) the DDE/D signature motif in their catalytic domains which corresponds to DD34D for mariner, DD34E for Tc1, DD37D for maT, DD37E, DD39D, and DD41D for rosa [8-10]. 
The mariner family, initially described in Drosophila mauritiana [11], is one of the best known elements belonging to this superfamily. This element is characterized by a patchy and large distribution among metazoans [12-14], which can be explained, in part, by horizontal transfer (HT), corresponding to its ability to transpose between genomes [15-17]. Due to the great diversity of this family, these elements are classified into several subfamilies based on phylogenetic studies. Five major distinct subfamilies including irritans, mauritiana, cecropia, mellifera/capitata, and elegans/briggsae were reported [12]. However, 16 minor subfamilies also exist with a more limited distribution [18-20]. Otherwise, the rosa monophyletic group, first identified in Ceratitis rosa and other Tephritid flies, is closely related to the mariner subfamilies $[9,16]$. Its main characteristic is a transposase with a DD41D motif, and the nucleotide identity between $M L E$ subfamilies is about 40 to $56 \%$ [12, 21].

While $M L E$ is characterized by a high proportion of inactive copies due to independent accumulation of substitution and indels, known as vertical inactivation [22], three elements, namely mos1, found in the fruit fly Drosophila mauritiana (mauritiana subfamily), Famar1 discovered in the common earwig Forficula auricularia (mellifera subfamily) and Mboumar9 isolated from the ant Messor bouvieri (mauritiana subfamily) are still naturally active, and thus able to be mobilized [12, 23-27]. Furthermore, the Himarl element from the horn fly Haematobia irritans (irritans subfamily) has been reconstructed by in vitro mutagenesis to restore a potential activity $[28,29]$. Due to their wide distribution and ability to successfully invade new genomes by horizontal transmission, naturally and artificially active mariner transposons are used as powerful molecular tools in transgenesis and insertional mutagenesis, inter alia leading to genetic control strategies of pests [29-32].

In aphid species, only a few studies have described the presence of mariner elements. For instance, (i) internal partial sequences of irritans and mellifera subfamilies were identified in vitro by a Polymerase Chain Reaction (PCR) amplification in the soybean aphid Aphis glycines [33], (ii) deleted sequences of mauritiana subfamily were characterized in seven fruit tree aphid species [34], (iii) in the first version of pea aphid Acyrthosiphon pisum genome [35], only three complete sequences, namely Mariner-Ap_1, 2 and 3, were published in RepBase [36]. However these sequences shared catalytic motif DD34E and should be more related to Tc1-elements.

Nowadays, three aphid's genomes are available in public databases. Indeed, the recent sequencing of the Russian wheat aphid Diuraphis noxia genome (Dnoxia_1.0 reference annotation release 101, http://www.ncbi.nlm.ih.gov) [37], the green peach aphid Myzus persicae genome (AphidBase, http://tools.genouest.org/tools/myzus/), and the new annotation of $A$. pisum genome (Acyr_2.0, new reference Annotation Release 102, http://www.ncbi.nlm.nih.gov/) offer an opportunity to investigate the diversity of the mariner family within and between aphid species, along with the evolutionary history and dynamics of these elements.

These species belong to the Macrosiphini tribe of the Aphididae family and diverged approximately 42.5 Mya [38]. They are found on different host plants: while M. persicae is generalist and found on peach trees or Solanaceae, A. pisum and D. noxia are specialist, infesting Fabaceae and cereals, respectively. In this paper, we explored these three genomes in order to identify mariner-related transposons and their non-autonomous derivatives through a homology-based method using as queries a panel of transposases from databases. Eleven TE clusters from A. pisum, seven from $D$. noxia and four from $M$. persicae have been detected. Classification and phylogenetic analysis suggested (i) that these lineages are divided into three groups: the irritans subfamily DD34D, rosa DD41D and a new group DD40/41D close to rosa and characterized by a long TIR, (ii) an evidence of vertical transfer with stochastic losses and several putative HT events. All these data provide new informations about the evolutionary history of these transposable elements in aphids.

\section{Methods \\ Supporting data}

The genome of Acyrthosiphon pisum and Diuraphis noxia are available at NCBI (http://www.ncbi.nlm.nih.gov/). The first contains $541 \mathrm{Mb}$ covering 23,925 scaffolds and the second includes $393 \mathrm{Mb}$ covering 5641 scaffolds [35, 37]. The genome of Myzus persicae, presenting $398 \mathrm{Mb}$ and spanning 34,598 scaffolds, is published in aphidbase (The International Aphid Genomics Consortium http://tools. genouest.org/tools/myzus/).

\section{Data mining}

A panel of 18 transposases sequences belonging to the five major mariner subfamilies DD34D and to the rosa DD41D group (Additional file 1) were used as queries in tBLASTN searches on the three aphid genomes, with default parameters. In order to determine the full sequence of each copy, the best hits were extracted with $5 \mathrm{~kb}$ flanking sequences and were manually investigated for TIR searches. Each new complete sequence was then used to retrieve more elements. Truncated copies located at the end of scaffolds and sequences less than $250 \mathrm{bp}$ were further discarded. The sequences closer to DDxE catalytic motif were excluded after a BLASTX search against transposases from this family. Finally, 115 sequences from $A$. pisum, 45 from $D$. noxia and 23 from M. persicae were obtained and used in this work. 


\section{Sequence analyses}

The nucleotide sequence analyses, including alignment, were done with the Aliview 1.18 [39]. USEARCH6.0 [40] was performed to cluster repetitive sequences using a threshold of $75 \%$ identity. Shorter copies flanked by two TIRs and with evidence of transposition (at least 2 copies) were considered as MITEs [4, 41]. Consensus sequences were derived using the relative majority rule.

The putative amino acid sequences were deduced by ExpasyTool (http://web.expasy.org/translate/) and then manually optimized. The nuclear localization sequence (NLS) and the helix-turn-helix (HTH) domain were searched using PSORTII [42] and GYM2.0 [43, 44], respectively (Additional file 2).

\section{Mining of available eukaryote genomes}

The complete nucleotide sequences previously identified were used in BLASTn searches against the nr (non-redundant nucleotide) and WGS (whole genome sequence) databases available on the NCBI. Sequences with more than $60 \%$ of nucleotide identity over more than $65 \%$ of the length of the query were extracted. These thresholds have been chosen to avoid recovering small fragments and sequences phylogenetically far from the subfamilies here considered. Cases of potential horizontal transfers between aphids and other taxa are considered when elements present more than $90 \%$ of identity covering more than $90 \%$ of the query sequences as proposed by several authors $[17,20]$.

\section{Classification and phylogenetic analysis}

The classification is based on the Unweighted Pair Group Method with Variation of Metric UPGM-VM [19], an ascending hierarchical classification analogous to the UPGMA method, with two main differences: (i) there is no arithmetical mean, the nucleotide sequences are aligned by pairs, (ii) the metric varies with the ascending classification and gap is considered as a fifth nucleotide. This variation allows a complete sequence to be gathered in the same group with the corresponding truncated and/or deleted sequences such as MITEs. Thus, the 183 elements extracted from aphid genomes were added to a set of 96 already known complete sequences from the Tc1-mariner-IS630 superfamily published in GenBank and to 50 sequences found in eukaryote genomes (Additional file 3). MITE classification is based on identity of TIRs, internal sequences of complete transposable elements and on the breakpoints of deletions.

For phylogenetic analysis, the amino acid sequences were aligned with Aliview1.18 [39] and the best-fitting ML model (AIC, matrix WAG + F + I + G) was selected using Protest 2.4 server [45]. Then, the phylogenetic analysis of transposases was computed using MEGA6 [46] with 1000 bootstrap replicates.

\section{Results}

Distribution and diversity of mariner and rosa elements within the Macrosiphini tribe

Search of sequences belonging to the main mariner subfamilies DD34D and to the rosa DD41D group was based on a homology approach (tBLASTN) using a set of 18 known transposases as queries (Additional file 1). We found a total of 115 copies from $A$. pisum clustered in 11 lineages, 45 from $D$. noxia clustered in seven lineages and 23 copies from $M$. persicae distributed in four lineages. A lineage corresponds to a group of sequences that is more than $75 \%$ similar and to clear phylogenetic clades (see below).

While 183 copies were extracted, 23 complete and potential autonomous sequences, representing $12.57 \%$ of all copies, have been identified in aphid genomes. A low copy number, ranging from one to six, per lineage and per species is observed. More precisely, only ten sequences distributed into nine lineages are found in $A$. pisum genome. All these sequences are named Apismar. For D. noxia, seven complete copies (Dnomar) are grouped into five lineages and only six copies from $M$. persicae (Mpmar) are gathered in the same group.

For most of these clusters (14 out of 15), the terminal inverted repeats (TIRs) necessary for transposition have been identified, as well as the TA target site duplication (TSD). The Apismar4.2 does not display a TSD. Interestingly, the whole nucleotide sequences appear heterogeneous in length. Some clusters with a short TIR (15-32 bp) have a full length of approximately $1.3 \mathrm{~kb}$ (i.e. Apismar1.2, Apismar4.1), while others (i.e. Apismar5.1, Apismar5.2) showed sizes longer than $2 \mathrm{~kb}$ due to long TIR sequences about 460 bp (Table 1).

Classification of the 183 aphid sequences, based on the 146 nucleotide sequences of the Tc1/mariner superfamily, was performed using a UPGM-VM method. This allows all sequences to be dealt with whatever their length, including the distantly related $T c 1$ and $T c 3$ sequences of animals, plants, fungi and bacteria like IS630 (Fig. 1, Additional file 3).

Results reveal that 75 copies (18 complete elements and 57 deleted/truncated sequences) belong to the irritans subfamily. They can be subdivided into three tribes: the first is widespread in aphids, namely Macrosiphinimar (Apismar1, Dnomar1 and Mpmar1). The second is close to known Batmar-like elements found in the bat Rhinolophus ferrumequinum genome. This group includes complete (Apismar2 and Dnomar2) and shorter sequences (deleted or truncated) from the three aphids species. The last tribe, namely Dnomar-like element, contains a complete copy from D. noxia (Dnomar3) and deleted/ truncated sequences from $D$. noxia and $M$. persicae.

Furthermore, two other groups can be identified: rosa DD41D and a new one close to the latter (Fig. 1, 
Table 1 Characteristics of 15 lineages corresponding to complete elements. The copy number, clade, length of the element, TIR and ORF, as well as the presence of potentially active copies, are specifically indicated for each complete sequence. The number of copies not truncated by " $\mathrm{N}$ " is mentioned in the fifth column. Potentially active copy = existence of at least copy with a complete ORF, with no frameshift or codon stop. In TIR sequences, the mirror sites are mentioned in bold

\begin{tabular}{|c|c|c|c|c|c|c|c|c|c|}
\hline \multirow[t]{2}{*}{ Clade } & \multirow[t]{2}{*}{ Tribe } & \multirow[t]{2}{*}{ Species } & \multirow{2}{*}{$\begin{array}{l}\text { Lineage } \\
\text { name }\end{array}$} & \multirow{2}{*}{$\begin{array}{l}\text { Complete } \\
\text { copy } \\
\text { number }\end{array}$} & \multirow{2}{*}{$\begin{array}{l}\text { Length } \\
\text { (bp) }\end{array}$} & \multicolumn{2}{|l|}{$\mathrm{TIR}$} & \multirow{2}{*}{$\begin{array}{l}\text { ORF } \\
\text { Length } \\
\text { (aa) }\end{array}$} & \multirow{2}{*}{$\begin{array}{l}\text { Potentially } \\
\text { active } \\
\text { copy }\end{array}$} \\
\hline & & & & & & $\begin{array}{l}\text { Length } \\
\text { (bp) }\end{array}$ & Sequences & & \\
\hline \multirow{10}{*}{$\begin{array}{l}\text { irritans } \\
\text { DD34D }\end{array}$} & \multirow{5}{*}{ Macrosiphinimar } & \multirow[t]{2}{*}{ A. pisum } & Apismar1.1 & 1 & 1334 & 28 & CGAGGCGTGTCCAGAAAGTAAGTGTACT & 354 & Yes \\
\hline & & & Apismar1.2 & 1 & 1317 & 15 & TTCGAAAAGTAAGGG & 355 & No \\
\hline & & \multirow[t]{2}{*}{ D. noxia } & Dnomar1.1 & 1 & 1347 & 28 & CGAGGCGTGTCCAGAAAGTAAGTGTACT & 354 & No \\
\hline & & & Dnomar1.2 & 1 & 1300 & 20 & TWCGAAAAKTAAGGGCCGTT & 347 & No \\
\hline & & M. persicae & Mpmar1.1 & 6 & 1334 & 28 & CGAGGCGTGTCCAGAAAGTAAGTGTACT & 355 & Yes \\
\hline & \multirow[t]{4}{*}{ Batmar-like } & \multirow[t]{2}{*}{ A. pisum } & Apismar2.1 & 1 & 1323 & 30 & CGAGGTATGACAATAAAATAAYGAGACTTT & 354 & Yes \\
\hline & & & Apismar2.2 & 2 & 1280 & 22 & AAYACCCAGACAAMAWKTATTA & 354 & No \\
\hline & & \multirow[t]{2}{*}{ D. noxia } & Dnomar2.1 & 2 & 1326 & 27 & YGAKGTGWSAMATAAAATAAACGAGAC & 357 & No \\
\hline & & & Dnomar2.2 & 2 & 1344 & 24 & CSWGGTGTGTTCAAAAAGWACYCG & 339 & No \\
\hline & Dnomar-like & D. noxia & Dnomar3.1 & 1 & 1360 & 26 & CGAGGGCGGGCTGATAAGTAATGCCT & 362 & No \\
\hline \multirow{3}{*}{$\begin{array}{c}\text { rosa } \\
\text { DD41D }\end{array}$} & \multirow{3}{*}{ Crmar2-like } & \multirow[t]{3}{*}{ A. pisum } & Apismar4.1 & 1 & 1355 & 32 & AAGGGTGTCTCAAAAAGAACGCCGGATTTRAA & 361 & Yes \\
\hline & & & Apismar4.2 & 1 & 1299 & 32 & GGGTTTTCAATARRAGCGCTCGAWSTTTSAT & 361 & No \\
\hline & & & Apismar4.3 & 1 & 1316 & 27 & GGTGCGGCAGAGCCRACTGACGAGTTT & 362 & Yes \\
\hline \multirow[t]{2}{*}{ LTIR } & DD41D & A. pisum & Apismar5.1 & 1 & 2307 & 466 & TCACCAATTTAGGGAACACTGAATTTCTCGGCT & 370 & Yes \\
\hline & DD40D & A. pisum & Apismar5.2 & 1 & 2423 & 460 & AATGTGTCAAACTTCTAGAGGTGTTTCTACACC & 351 & No \\
\hline
\end{tabular}

Additional file 3). rosa DD41D is represented by 44 copies restricted to $A$. pisum (Apismar4) and D. noxia genomes. They are clustered with Crmar2 found in the Diptera Mediterranean fruit fly Ceratitis rosa. The second group, characterized by a long TIR, named LTIR-like elements, mainly comprises sequences from the pea aphid (Apismar5.1, Apismar5.2) and may correspond to a new subfamily.

In the same genome, at least four lineages can coexist. However, large differences are observed among species (Fig. 1). Indeed, in $M$. persicae, a potential autonomous element (Mpmar1) from Macrosiphinimar, related to short sequences, is identified. No rosa elements are detected and only deleted/truncated copies belonging to LTIR-like, Dnomar-like and Batmar-like elements are detected. In $D$. noxia, five irritans lineages are found. They include potential autonomous elements (Dnomar) and a few deleted/truncated copies of the same lineage. Two lineages are composed by short sequences belonging to rosa and LTIR clades. Furthermore, the genome of $A$. pisum is free of Dnomar-like elements. The other lineages are mainly represented by deleted/truncated copies and only a few complete sequences (Apismar1-5) can be detected. Hence, the large diversity of these elements among species may reflect the independent evolutionary history of these lineages or specific properties of the genome.
TIRs show a higher degree of identity in the irritans subfamily, suggesting a possible recent common ancestor, while they seem to be less conserved in rosa and LTIR elements (Additional file 4). In addition, TIRs do not present palindromic motifs, but only mirror repeats can be detected in Apismar2.1 and Dnomar2.1 belonging to Batmar- like elements (Table 1).

Otherwise, the screening of NCBI-nr and WGS databases (Eukaryotes) with the complete elements identified in aphid's genomes reveals only one sequence having a level of similarity above 90\%, with cover queries up to $90 \%$. In fact, it concerns a complete element belonging to the irritans subfamily found in the genome of the Coleoptera Agrilus planipennis, which is closely related to Dnomar 2.2 from $D$. noxia with $92 \%$ of similarity (Fig. 1, Additional file 3).

\section{Protein and phylogenetic analyses}

The protein sequences of the 15 full clusters are characterized by an ORF encoding about 339 to 370 aa (Fig. 2, Table 1 and Additional file 2). They are aligned with 56 other copies of the Tc1-mariner superfamily belonging to non-aphids species. The topology of the ML phylogenetic tree is roughly similar to the classification based on nucleotide sequences (Fig. 1, Additional file 3). Indeed, the five tribes, previously described, are supported by high bootstrap values (98-100\%). The percentage of 


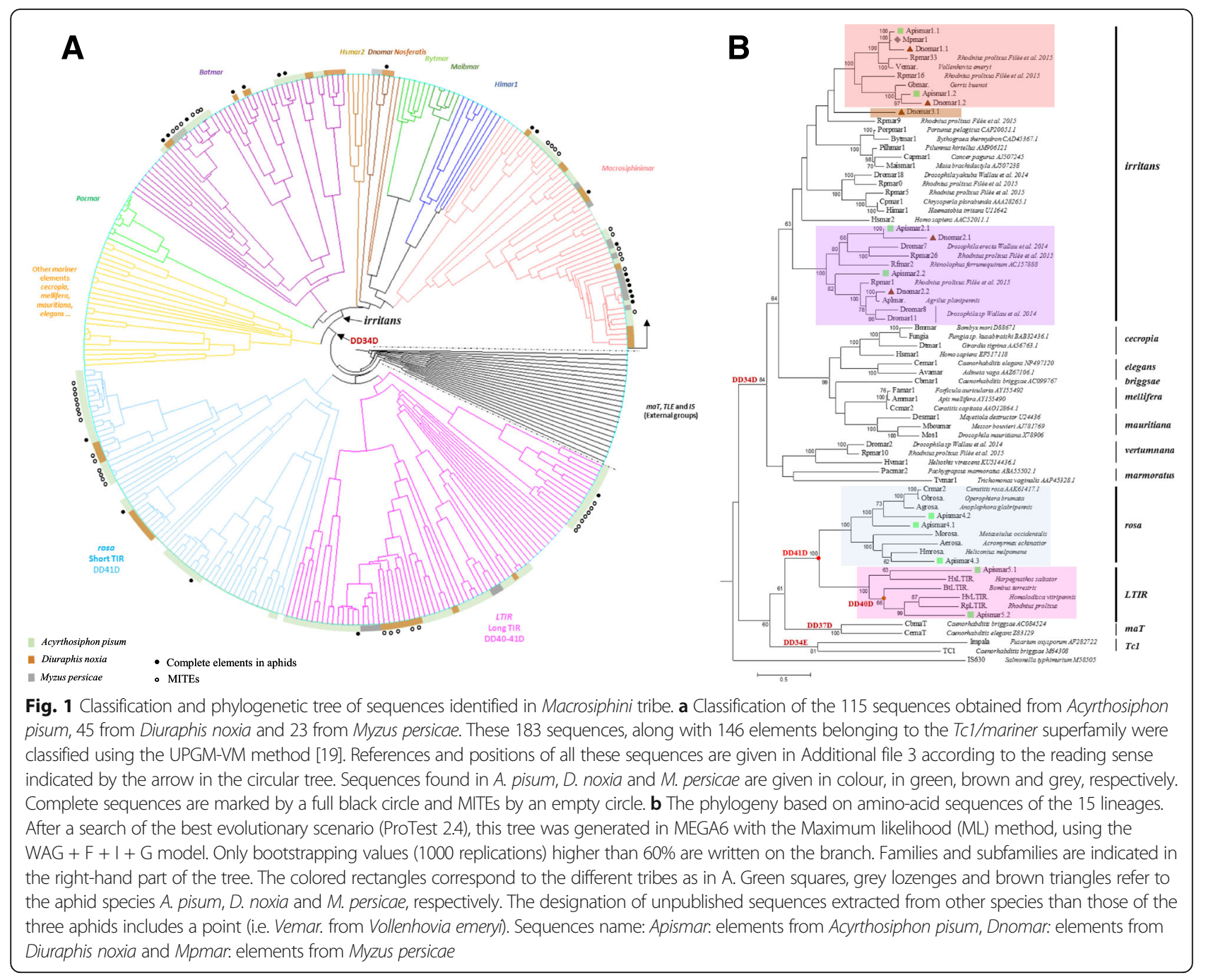

identity between these clades varies from 28 to $59 \%$ (Additional file 5).

Only six complete sequences (Apismar1.1, Mpmar1, Apismar2.1, Apismar4.1, Apismar4.3 and Apismar5.1) present an intact ORF with no frameshift or codon stop, suggesting that they might be active (Table 1, Additional file 2). An analysis of the transcriptomes of the two species ( $A$. pisum and $M$. persicae) was performed using these 6 sequences with a complete ORF. Five sequences (Apismar1.1, Mpmar1.1, Apismar4.1, Apismar4.3 and Apismar5.1) present a full-length transcript, while the last one Apismar2.1 presents an internal deletion leading to the loss of 140 aa. The sequences related to the conserved motifs, especially WVPHEL and YSPDLA, as well as the catalytic site DD34D considered as the mariner signature $[47,48]$, are detected in most of the sequences belonging to the irritans subfamily: Macrosiphinimar, Batmar-like elements and Dnomar-like elements (Fig. 2, Additional file 2). The less conserved motif is WVPHEL, localized between the HTH motif and the first D. The catalytic site is relatively well conserved (7 out of 10) with a length polymorphism between the three residues. Two sequences are deprived of HTH and one of NLS. These three copies are probably inactive.

In the rosa clade, close to Crmar2-like elements, the catalytic domain is DD41D rather than the canonical DD34D (Fig. 2, Additional file 2). While the NLS motif is lacking, the HTH is located from position 88 to 110 in Apismar4.1 and from 90 to 112 in Apismar4.3.

The classification and phylogenetic tree showed the presence of a monophyletic clade related to rosa DD41D (43\% \pm 0.016 of similarity), designated LTIR. This monophyletic group, characterized by long sequences (> $2.3 \mathrm{~kb}$ ) with a long TIR (> $460 \mathrm{bp}$ ), can be divided into two tribes based on the transposase similarities. The NLS motif is absent and in the catalytic domain the distance between the second and the third D is of 40 aa for Apismar5.2 and 41 aa for Apismar5.1. Otherwise, HTH 


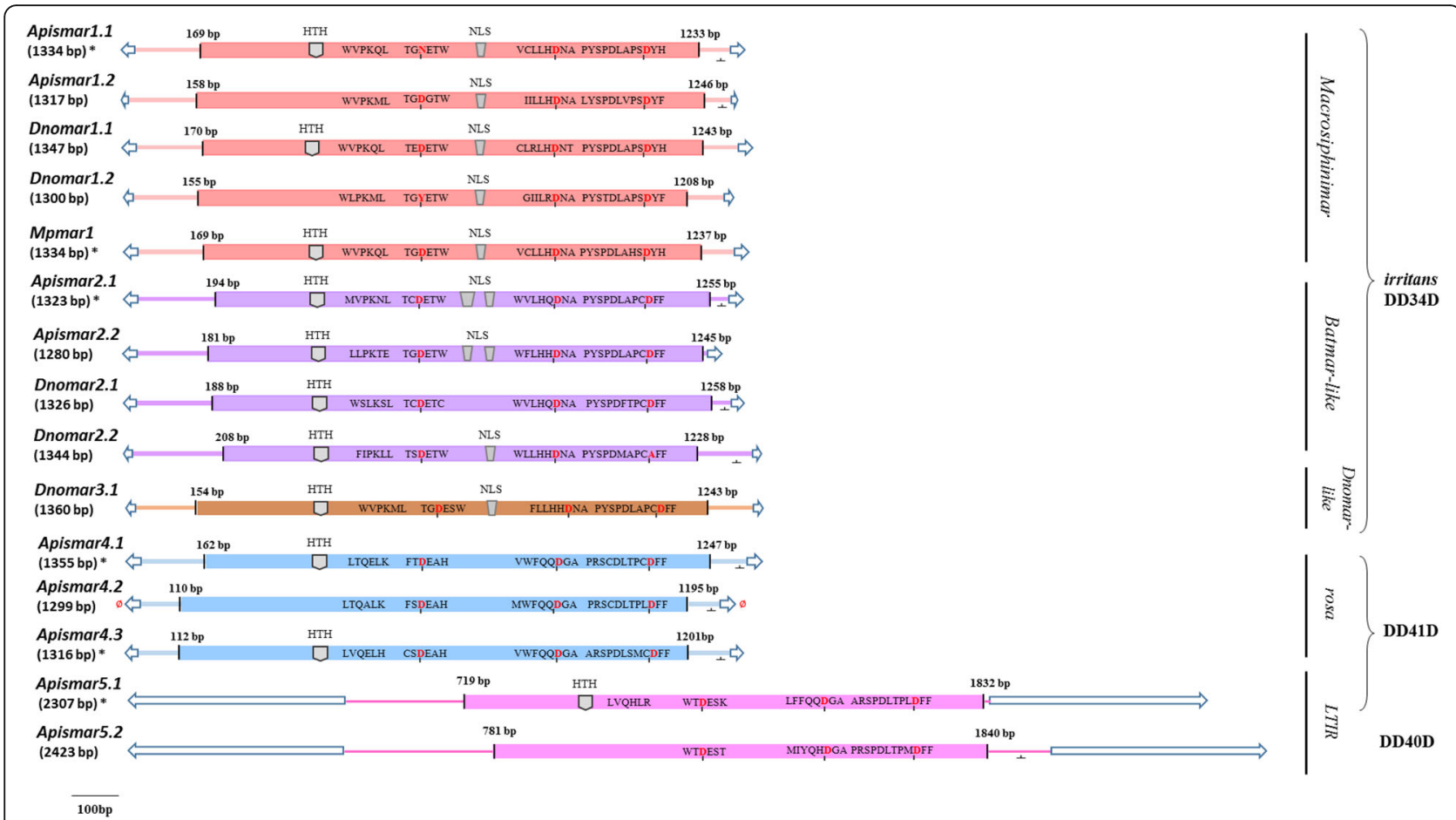

Fig. 2 Schematic representation of the 15 lineages corresponding to complete sequences found in aphid's genomes. The elements are arranged and colored (as in Figure 1) according to the clades they belong to. Potentially active copies are marked with asterisks. The lack of TA (TSD) is marked by a slashed zero in red. Blue arrows indicate TIR, while bold lines represent UTRs. A turned T shows the presence of polyAdenylation site "AATAAA". In transposase gene, the three catalytic residues containing aspartic amino acids marked in red are indicated. The helix turn helix $(\mathrm{HTH})$ region, the nuclear localization signal (NLS), and motifs related to WVPHEL are also mentioned. Sequences name: Apismar: elements from Acyrthosiphon pisum, Dnomar: elements from Diuraphis noxia and Mpmar: elements from Myzus persicae

motif is only present in LTIR DD41D (Fig. 2, Additional file 2). The phylogenetic tree also indicated that rosa DD41D and LTIR DD40-41D elements are closer to $m a T$ and $T c 1$ than to mariner subfamilies (Fig. 1). The comparison of the sequences surrounding the catalytic site is summarized in Fig. 3. The flanking sequences of the second $\mathrm{D}$ is clearly distinct between the different groups (rosa/LTIR/maT vs the mariner subfamilies).

\section{MITEs occurrence: Structure and evolution}

MITEs are defined as short non-autonomous copies which are known to derive from autonomous ones. They do not encode functional transposase but can be transmobilized thank to the transposase of complete copies.

MITEs, detected in the present work, represent 43 copies i.e. $23.5 \%$ of all extracted sequences. Only the Dnomar-like tribe is free of MITEs (Table 2). For the others, there is a large-size polymorphism, and MITEs are clustered into 11 sublineages based on the breaking points of the main internal deletion and the TIR sequences. All of these sequences, except one (MITE1.1 sub2), can be related to a full-length copy (Figs. 1 and 4 and Additional file 3). Microhomologies have been found at the breaking points of the internal deletions for most of the MITEs. According to the nomenclature proposed by Negoua et al. [49], they are of the BPEE type for seven sublineages of MITE, and of the BPNN type for two other sublineages (Table 2). For the remaining (MITE1.1) no microhomology can be detected.

In the irritans clade, represented by the Macrosiphinimar tribes and Batmar-like elements, only A. pisum and $M$. persicae contain MITEs, with size varying between 908 and $1165 \mathrm{bp}$. The first tribe (MITE1.1) includes nine copies from the pea aphid clustered in two sublineages (sub1 and sub2) which only share the first 12 nucleotides of the TIRs. An additional lineage (MITE1.2), closely related to MITE1.1sub1, is found in M. persicae. These two sublineages present similar TIRs and an average identity of $81.8 \%$. However, they do not have similar breaking points (Fig. 4). These two types of MITEs are related to putative autonomous copies found in each species (Apismar1.1 and Mpmar1.1 respectively) showing $99 \%$ of identity.

A similar situation is observed for the rosa clade when MITE4.1sub1 and MITE4.2 are compared. The MITE4.1 lineage, includes 12 copies with lengths from 349 to 548 bp, comprised two sublineages. Although clearly related, these sublineages seemed to result from 


\begin{tabular}{|c|c|c|c|c|c|}
\hline & & & D & D & $D / E$ \\
\hline \multirow{5}{*}{$\begin{array}{c}\text { rosa } \\
\text { DD41D }\end{array}$} & Crmar2 & $:$ & FSDEA & QDGA & SCDLTPLDF \\
\hline & Apismar4.1 & : & FTDEA & QQDGA & SCDLTPCDF \\
\hline & Apismar4.2 & : & FSDEA & QQDGA & SCDLTPLDF \\
\hline & Aerosa. & : & FSDEA & QDGA & SPDLTPCDF \\
\hline & Apismar4.3 & : & CSDEA & $Q Q D G A$ & SPDLSMCDF \\
\hline \multirow{4}{*}{$\begin{array}{c}L T I R \\
\text { DD40-41D }\end{array}$} & BtLTIR. & : & WTDER & $Q Q D G A$ & SPDLTPLDF \\
\hline & HasLTIR. & : & FSDEA & $Q Q D G A$ & SPDLTSLDF \\
\hline & Apismar5.1 & : & WTDES & QQDGA & SPDITPLDF \\
\hline & Apismar5.2 & : & WTDES & QHDGA & SPDLTPMDF \\
\hline \multirow{2}{*}{$\begin{array}{c}\text { maT } \\
\text { DD37D }\end{array}$} & CemaT & $:$ & FTDEK & QQDGA & SPDLNPMDY \\
\hline & CbmaT & : & WTDEK & QQDWA & SPDLNPMDF \\
\hline \multirow{2}{*}{$\begin{array}{c}T c 1 \\
\text { DD34E }\end{array}$} & Impala & $:$ & WSDEC & MHDNA & SPDLNPIE- \\
\hline & $\mathrm{CbTc1}$ & : & FSDES & QQDND & SPDLNPIE- \\
\hline \multirow{16}{*}{$\begin{array}{c}\text { mariner } \\
\text { subfamilies } \\
\text { DD34D }\end{array}$} & Mos1 & : & TGDEK & LHDNA & SPDLAPSDY \\
\hline & Hsmar1 & : & TCDEK & LHDNA & SPDLSPTDY \\
\hline & Cemar1 & : & TGDEK & LHDNA & SPDLAPTDY \\
\hline & Famar1 & : & TGDEK & HHDNA & SPDLAPSDY \\
\hline & Cpmar1 & $:$ & TMDET & HQDNA & SPDLAPSDF \\
\hline & Dnomar3.1 & : & TGDES & HHDNA & SPDLAPCDF \\
\hline & Rfmar2 & : & TGDET & HHDNA & SPDLAPNDF \\
\hline & Apismar2.1 & : & TCDET & HQDNA & SPDLAPCDF \\
\hline & Dnomar2.1 & : & TCDET & HQDNA & SPDFTPCDF \\
\hline & Dnomar2.2 & : & TSDET & HHDNA & SPDMAPCAF \\
\hline & Apismar 2.2 & : & TGDET & HHDNA & SPDLAPCDF \\
\hline & Apismar1.1 & $:$ & TGNET & LHDNA & SPDLAPSDY \\
\hline & Dnomar1.1 & : & TEDET & LHDNT & SPDLAPSDY \\
\hline & Mpmar1 & $:$ & TGDET & LHDNA & SPDLAHSDY \\
\hline & Apismar1.2 & : & TGDGT & LHDNA & SPDLVPSDY \\
\hline & Dnomar1.2 & & TGYET & LRDNA & STDLAPSDY \\
\hline
\end{tabular}

Fig. 3 Multiple alignments of catalytic motifs of TC1, mariner, maT families with the 15 lineages identified in aphids

independent internal deletions of the Apismar4.1 complete element. The $D$. noxia genome contains two copies of a MITE of 578 bp (MITE4.2) which are also closely related to the autonomous element Apismar4.1 (Fig. 4).

For the LTIR DD41D tribe, MITE5.1, only found in D. noxia, comprises five copies (790-822 bp) with the same breakpoints, and are related to the autonomous element Apismar5.1. No MITE5.1 was retrieved in the A. pisum genome. Furthermore, MITE5.2 of LTIR DD40D tribe identified in the pea aphid is composed of seven short copies (411 and $441 \mathrm{bp}$ ). They are divided into two sublineages depending on the breakpoint positions, probably resulting from independent internal deletions (Fig. 4).

Globally, these results show that (i) MITEs in aphid species are less frequent than in Drosophila ananassae (about 240 copies) [41] and in Rhodnius prolixus (about 400 copies) [20]; (ii) irritans clades do not generate MITEs smaller than $900 \mathrm{bp}$, in contrast to rosa and LTIR-like elements clades; (iii) three MITE sublineages (MITE2.2, MITE4.2 and MITE5.1) are closely related to autonomous copies found in other species; (iv) orphan MITE sublineages can be detected with no full-length partner (MITE1.1 sub2). In the later case, it cannot be excluded that active copies still exist in other populations or closely related species.

The distribution of MITEs and their relationship with full-length elements show that their phylogeny is inconsistent with that of the species. Several scenarios involving the existence of ancestral polymorphism, current population polymorphism (presence/absence of autonomous copies and/or MITEs), stochastic loss of autonomous copies and/or horizontal transfers can be proposed.

To infer the dynamics of MITEs identified in the aphid genomes, we generated consensus sequences for each sublineage in order to estimate their period of amplification from their percentage of divergence, as proposed by Le Rouzic et al. [50] and Wallau et al. [41]. Except for two sequences of the MITE4.1 sub2 showing 69 and $72 \%$ of identity with the consensus of this lineage, all others exhibit a level of identity higher than $85 \%$ (Fig. 5). While the transposition rate (trans-mobilization) of these copies is unknown, we observed that some of them are almost identical (97-99\% of identity) suggesting that these copies are still trans-mobilizable or were recently inactivated. The remaining sequences (identity level from $85 \%$ to $95 \%$ ) are less conserved and probably correspond to ancient trans-mobilization, and are no longer mobilizable.

\section{Discussion}

The three species of aphids, A. pisum, D. noxia and $M$. persicae, present different genome sizes $(541 \mathrm{Mb}$, $393 \mathrm{Mb}$ and $398 \mathrm{Mb}$ respectively), which correspond to different TE equipment $[35,37]$, i.e. $38 \%$ and $11.5 \%$ for the first two species (no information being available for $M$. persicae), suggesting as previously proposed that the contribution of TEs to genome size variation is greater relative to other sources of variation $[41,51,52]$.

In the present work, we focused on a survey of $M L E$-related elements in aphid genomes. Our data are in agreement to the previous observation since a total of 115, 45 and 23 sequences, extracted from $A$. pisum, D. noxia and $M$. persicae, respectively, are clustered into 22 lineages. The relative abundance of $M L E$-related elements in these three aphids' genomes is low compared to other insect genomes. For instance, mariner subfamilies are represented by 10,836 copies in the $700 \mathrm{Mb}$ genome of the Hemiptera Rhodnius prolixus [20] and 642 copies in the $156 \mathrm{Mb}$ genome of the Drosophila eugracilis [41]. Otherwise, the Tc1-mariner superfamily is poorly represented in each aphid genome compared to other superfamilies of DNA transposons, such as piggyBac or hAT (personal data). This observation might be an illustration of the competition that may occur between 


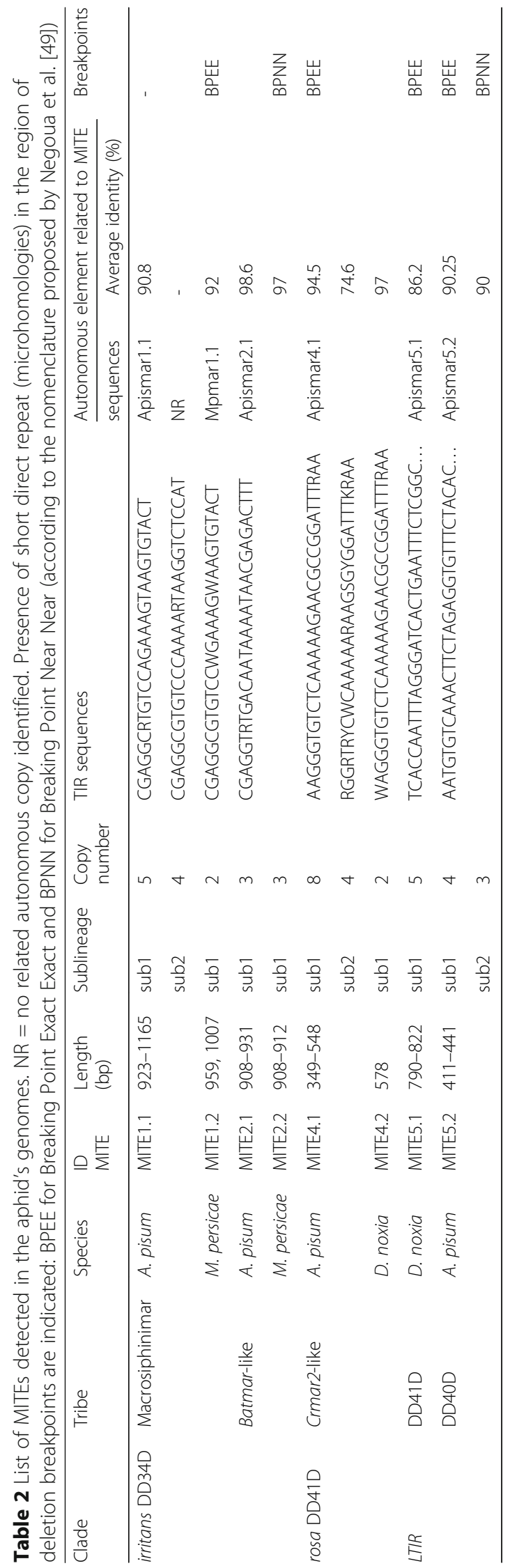



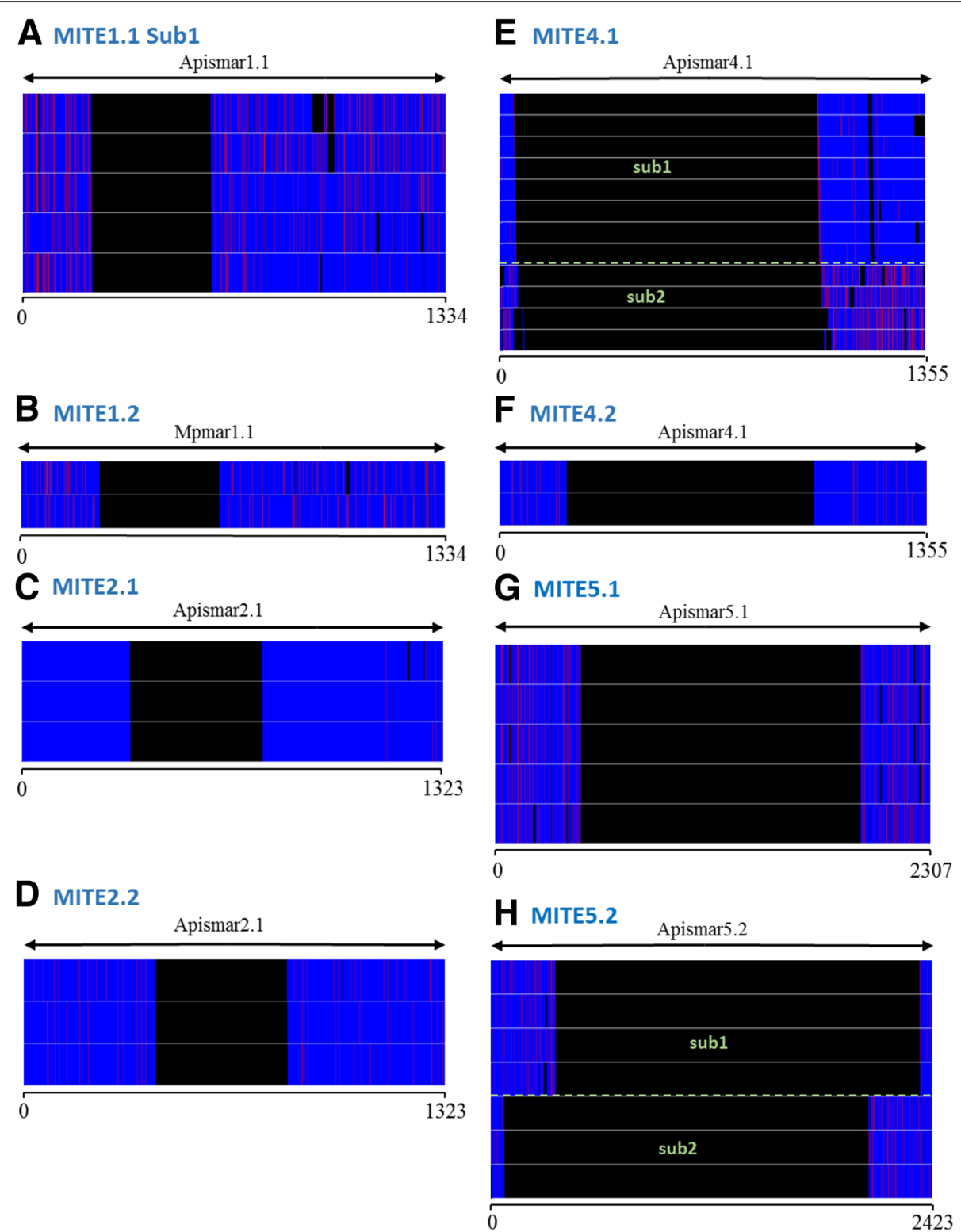

Fig. 4 Sequence alignments of MITE lineages with a longer autonomous partner. For each alignment (a-h), sequences are in blue, showing substitutions in red and gaps in black. The autonomous copies related to MITE and the global structure of the copies are shown on top, with arrowheads corresponding to TIR. Similar copies in length and sequence-defined sublineages (numbered in green). Given the lack of homology with the full potential element, MITE1.1 sub2 is not represented. $\mathbf{a}, \mathbf{c}, \mathbf{e}$ and $\mathbf{h}$ are found in A. pisum, $\mathbf{b}$ and $\mathbf{d}$ in M. persicae, $\mathbf{f}$ and $\mathbf{g}$ in D. noxia

superfamilies as described by Abrusán and Krambeck [53]. However, today without a complete and detailed overview of TE equipment of these genomes, we do not have strong arguments to conclude that such a result is due to competition.

In the mariner family, only members of the irritans subfamily are identified in the aphid's genomes. They belong to the Macrosiphinimar, Batmar-like and Dnomar-like tribes, and are characterized by the DD34D catalytic site. Moreover, only three lineages might still be active (Apismar1.1, Mpmar1.1 and Apismar2.1). No sequence related to other mariner subfamilies (i.e. mauritiana, mellifera, cecropia, elegans) is found in these genomes, although they have been identified in vitro in other species belonging to a closely related aphid species such as Aphis glycines [33] and seven tree aphids [34].

However, sequences belonging to the rosa family (initially closely related to the mariner family [9]) have been detected in A. pisum and D. noxia; and a novel clade (LTIR-like) has been identified. Such LTIR elements including the DD41D motif, designated as Lsra transposons, were described by Zhang et al. [54]. 


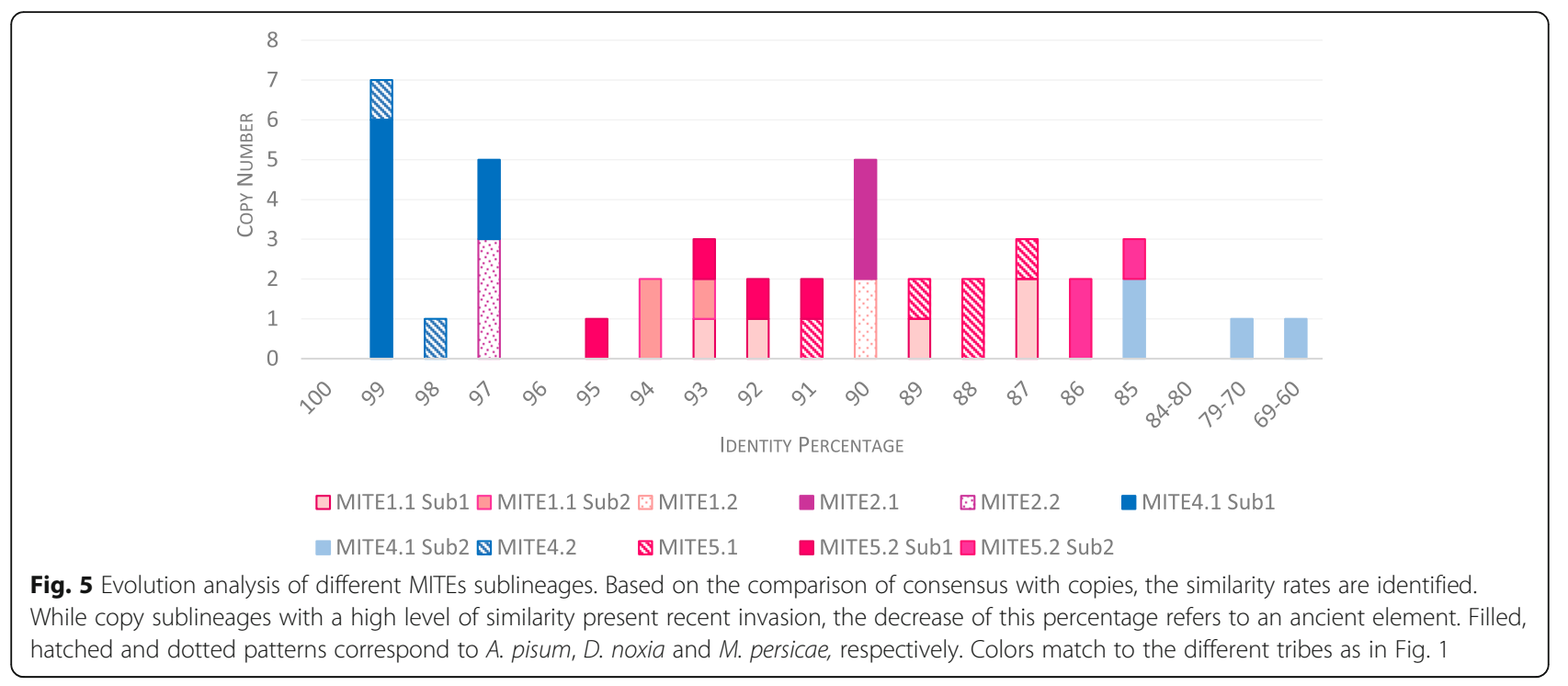

This clade is closely related to the rosa subfamily but is characterized by long TIRs (about 460 bp vs 28$32 \mathrm{bp}$ ). Moreover, conservation of some specific amino acid residues in their catalytic region, especially the final aspartic acid (D) rather than glutamic acid (E), and phylogenetic analysis revealed that rosa and LTIR-like elements are more closely related to maT elements than to $T c 1$ and mariner ones. Therefore, we suggest that rosa DD41D and LTIR-like elements constitute a large new family belonging to Tc1/mariner.

Distribution, diversity and phylogeny of these elements in the three aphids' genomes are probably the result of vertical transmissions associated to an ancestral polymorphism. In such a situation, closely related sequences derived from the same ancestral copy can be found in several species, while copies derived from different ancestral copies and found in the same genome, can be more distantly related (see for instance [55-57]). Host genomes are also able to repress TE activity [58, 59], leading to their elimination by stochastic loss or vertical extinction. Therefore, the absence of members of the rosa family may be due to a stochastic loss during the evolutionary trajectory of $M$. persicae. A similar observation was illustrated in some Drosophila species for mariner subfamilies $[41,60]$.

The high level of similarity between MITEs and autonomous partner indicates that short sequences are internally deleted elements, deriving from complete copies. Most of them exhibit direct repeat microhomologies exactly (BPE) or nearly (BPN) to the deletion breakpoints, suggesting that these internal deletions are probably due to abortive gap repair [49, 61, 62]. However, MITEs and related complete copies can be found in two different species, as described in the $R$. prolixus and Drosophila genus [20, 41]. This is the case for MITE2.2, MITE4.2 and MITE5.1. To explain such observations, two scenarios can be proposed. First, the ancestral autonomous element at the origin of MITEs may have been lost after the MITE amplification, but was maintained in another species. Another hypothesis consists in the emergence of MITEs after internal deletion(s) of a complete copy, these MITEs being then mobilized by the transposase of another copy closely related to the first one.

Finally, horizontal transfer may also occur for all these sequences between distantly related species. For instance, the mariner autonomous transposon Dnomar2.2 from $D$. noxia is closely related to the sequence of Agrilus planipennis. Despite a divergence time of about 361 Mya between these two species (http://www.timetree.org/ home), the phylogenetic tree of these elements is inconsistent with that of the species. Moreover, HT could also explain the patchy distribution of MITE elements in aphids. However, in all these cases, the transfer mechanism(s) remain unknown and only propositions are suggested, like those proposed in Silva et al. [63] and Loreto et al. [64].

\section{Conclusion}

Our results represent the first in silico evidence of diversity and possible evolutionary scenarios of elements belonging to the three clades: irritans, rosa and a new one named LTIR-like elements in aphid genomes. This latter clade is characterized by long TIRs and subdivided into two distinct subgroups based on the catalytic domain signature DD40D or DD41D. Moreover, based on protein and phylogenetic analyses, the rosa and LTIR transposons are related to 
maT DD37D elements, indicating a recent common ancestor. We also demonstrated the presence of several MITE lineages deriving from internal deletion of autonomous elements. Finally, this study proposes an update of the classification of the Tc1/mariner superfamily. Data analyses will offer a basis for future research aiming to understand the role of transposable elements during evolution and to develop biotechnological applications for the genetic control of aphid species.

\section{Additional files}

Additional file 1. mariner and rosa transposases sequences used as queries in the tBLASTN search (Species, Clades, Accession number). (PDF $408 \mathrm{~kb}$ )

Additional file 2. Amino acid sequences of the transposase of the 15 complete elements. The three aspartic residues of the catalytic domain are marked in red, the sequences related to the WVPHEL-specific motif of the DNA binding domain are indicated in black bold as well as the helix turn helix (HTH) region (underlined) and the NLS (in blue). Stop codons are represented by asterisks $(*$ ). (PDF $425 \mathrm{~kb}$ )

Additional file 3. Sequences classified by UPGM-VM method according to the reading sense indicated by the arrow in the circular tree. Deleted or truncated sequences are indicated by an asterisk (*). (PDF $276 \mathrm{~kb}$ )

Additional file 4. TIR sequences for each clade. TIR consensus per clade was generated using the Web-Logo server (http://weblogo.berkeley.edu/ logo.cgi). At each position the nucleotides are stacked one on top of another with the most frequent one on the top. It displays the frequency of bases at each position, with height indicating the proportion of occurrence. The vertical scale is in bits with maximum of two bits possible at each position, indicating that there can be possibility of four different bases at each position. For LTIR- like elements, only the first 57 nucleotides are presented. (PDF $111 \mathrm{~kb}$ )

Additional file 5. Pairwise divergence matrix between amino acid lineages. Fifteen complete sequences have been aligned using Aliview, The alignment was then transferred in GENEDOC software [65] to obtain the identity percentage. Sequences name: Apismar: elements from Acyrthosiphon pisum, Dnomar: elements from Diuraphis noxia and Mpmar: elements from Myzus persicae. (PDF $354 \mathrm{~kb}$ )

\section{Abbreviations}

BPEE: Breaking point exact exact; BPNN: Breaking point near near; HTH: Helix turn helix; LTIR-like elements: Long terminal inverted repeats like elements; MITES: Miniature inverted-repeat transposable elements; MLE: mariner-like element; NCBI-nr: Non-redundant nucleotide; NLS: Nuclear localization signal; TE: Transposable element; TIR: Terminal inverted repeats; TLE: Tc7-like element; TSD: Target site duplication; UPGM-VM: Unweighted pair group method with variation of metric; WGS: Whole genome sequence

\section{Acknowledgements}

Authors thank Aurélie Hua-Van for its helpful comments and Malcolm Eden for the English review of the manuscript.

\section{Funding}

This work was financially supported by the Tunisian Ministry of Higher Education and Scientific Research, the University of Tunis El Manar, the Centre National de la Recherche Scientifique and the Paris-Sud University.

Availability of data and materials

All the data supporting these findings is contained within the manuscript.

\section{Authors' contributions}

$M B, M M$ and $P C$ conceived and designed research. MB performed research. $M B, J F, I K, M M K$ and JDR contributed to the analysis. MB, MM and PC drafted the manuscript. All authors have read and approved the final manuscript.

\section{Competing interests}

The authors declare that they have no competing interests.

Consent for publication

Not applicable

Ethics approval and consent to participate

Not applicable

\section{Publisher's Note}

Springer Nature remains neutral with regard to jurisdictional claims in published maps and institutional affiliations.

\section{Author details}

'Laboratoire Evolution, Génomes, Comportement, Ecologie CNRS, Université Paris-Sud, IRD, Université Paris-Saclay, 1 avenue de la Terrasse, 91198 Gif-sur-Yvette Cedex, France. ${ }^{2}$ Faculté des Sciences de Tunis, UR11ES10 Génomique des Insectes Ravageurs de Cultures, Université de Tunis El Manar, 1002 Tunis, Tunisie.

Received: 4 January 2017 Accepted: 9 June 2017

Published online: 29 June 2017

\section{References}

1. Biémont C, Vieira C. Genetics: Junk DNA as an evolutionary force. Nature. 2006:443(7111):521-4

2. Feschotte C, Pritham EJ. DNA transposons and the evolution of eukaryotic genomes. Annu Rev Genet. 2007:41:331-68.

3. Chénais B, Caruso A, Hiard S, Casse N. The impact of transposable elements on eukaryotic genomes: From genome size increase to genetic adaptation to stressful environments. Gene. 2012;509(1):7-15.

4. Feschotte C, Zhang X, Wessler SR. Miniature inverted-repeat transposable elements (MITEs) and their relationship with established DNA transposons In: Craig NL, Craigie R, Gellert M, Lambowitz AM, editors. In Mobile DNA II. Washington DC: ASM Press; 2002. p. 1147-58.

5. Brillet B, Bigot $Y$, Augé-Gouillou C. Assembly of the Tcl and mariner transposition initiation complexes depends on the origins of their transposase DNA binding domains. Genetica. 2007;130(2):105-20.

6. Plasterk RH, Izsvák Z, Ivics Z. Resident aliens: The Tc1/mariner superfamily of transposable elements. Trends Genet. 1999;15(8):326-32.

7. Lohe AR, Hartl DL. Autoregulation of mariner transposase activity by overproduction and dominant-negative complementation. Mol Biol Evol. 1996;13(4):549-55.

8. Shao H, Tu Z. Expanding the diversity of the IS630-TC1-mariner superfamily: Discovery of a unique DD37E transposon and reclassification of the DD37D and DD39D transposons. Genetics. 2001;159(3):1103-15.

9. Gomulski LM, Torti C, Bonizzoni M, Moralli D, Raimondi E, Capy P, et al. A new basal subfamily of mariner elements in Ceratitis rosa and other tephritid flies. J Mol Evol. 2001;53(6):597-06.

10. Claudianos C, Brownlie J, Russell R, Oakeshott J, Whyard S. maT a clade of transposons intermediate between mariner and Tc1. Mol Biol Evol. 2002:19(12):2101-9.

11. Medhora MM, MacPeek AH, Hartl DL. Excision of the Drosophila transposable element mariner: Identification and characterization of the Mos factor. EMBO J. 1988;7(7):2185.

12. Robertson HM, MacLeod EG. Five major subfamilies of mariner transposable elements in insects, including the Mediterranean fruit fly, and related arthropods. Insect Mol Biol. 1993;2(3):125-39.

13. Auge-Gouillou C, Bigot Y, Pollet N, Hamelin MH, Meunier-Rotival M, Periquet G. Human and other mammalian genomes contain transposons of the mariner family. FEBS Lett. 1995;368(3):541-6.

14. Sinzelle L, Chesneau A, Bigot Y, Mazabraud A, Pollet N. The mariner transposons belonging to the irritans subfamily were maintained in chordate genomes by vertical transmission. J Mol Evol. 2006;62(1):53-65. 
15. Laha T, Loukas A, Wattanasatitarpa S, Somprakhon J, Kewgrai N, Sithithaworn P, et al. The bandit, a new DNA transposon from a hookworm-possible horizontal genetic transfer between host and parasite. PLoS Negl Trop Dis. 2007;1(1):e35.

16. Dupeyron M, Leclercq S, Cerveau N, Bouchon D, Gilbert C. Horizontal transfer of transposons between and within crustaceans and insects. Mob DNA. 2014;5:4

17. Wallau GL, Capy P, Loreto E, Le Rouzic A, Hua-Van A. VHICA, a new method to discriminate between vertical and horizontal transposon transfer: Application to the mariner family within Drosophila. Mol Biol Evol. 2016;33(4):1094-09.

18. Robertson HM, Soto-Adames FN, Walden KK, Avancini RM, Lampe D. The mariner transposons of animals: Horizontally jumping genes. In: Kado $\mathrm{Cl}$, editor. Horizontal gene transfer. San Diego CA: Academic Press; 2002. p. 173-85.

19. Rouault JD, Casse N, Chénais B, Hua-Van A, Filée J, Capy P. Automatic classification within families of transposable elements: Application to the mariner family. Gene. 2009;448(2):227-32.

20. Filée J, Rouault JD, Harry M, Hua-Van A. Mariner transposons are sailing in the genome of the blood-sucking bug Rhodnius prolixus. BMC Genomics. 2015;16(1):1.

21. Bigot Y, Brillet B, Auge-Gouillou C. Conservation of palindromic and mirror motifs within inverted terminal repeats of mariner-like elements. J Mol Biol. 2005;351(1):108-16.

22. Kidwell MG, Lisch DR. Perspective: Transposable elements, parasitic DNA, and genome evolution. Evolution. 2001;55(1):1-24.

23. Jacobson JW, Medhora MM, Hartl DL. Molecular structure of a somatically unstable transposable element in Drosophila. Proc Natl Acad Sci U S A. 1986;83(22):8684-8.

24. Maruyama K, Hartl DL. Evidence for interspecific transfer of the transposable element mariner between Drosophila and Zaprionus. J Mol Biol. 1991;33(6):514-24.

25. Medhora M, Maruyama K, Hartl DL. Molecular and functional analysis of the mariner mutator element mos1 in Drosophila. Genetics. 1991;128(2):311-8.

26. Barry EG, Witherspoon DJ, Lampe DJ. A bacterial genetic screen identifies functional coding sequences of the insect mariner transposable element Famar1 amplified from the genome of the earwig, Forficula auricularia. Genetics. 2004;166(2):823-33.

27. Muñoz-López M, Siddique A, Bischerour J, Lorite $P$, Chalmers R, Palomeque T. Transposition of Mboumar-9: Identification of a new naturally active mariner-family transposon. J Mol Biol. 2008;382(3):567-72.

28. Lampe DJ, Akerley BJ, Rubin EJ, Mekalanos JJ, Robertson HM. Hyperactive transposase mutants of the Himar1 mariner transposon. Proc Natl Acad Sci U S A. 1999;96(20):11428-33.

29. Rholl DA, Trunck LA, Schweizer HP. In vivo Himar1 transposon mutagenesis of Burkholderia pseudomallei. Appl Environ Microb. 2008;74(24):7529-35.

30. O'brochta DA, Atkinson PW. Transposable elements and gene transformation in non-drosophilid insects. Insect Biochem Mol Biol. 1996;26(8):739-53.

31. Wang W, Swevers L, latrou K. mariner (mos 1) transposase and genomic integration of foreign gene sequences in Bombyx mori cells. Insect Mol Biol. 2000;9(2):145-55.

32. Delaurière L, Chénais B, Hardivillier Y, Gauvry L, Casse N. mariner transposons as genetic tools in vertebrate cells. Genetica. 2009;137(1):9-17.

33. Mittapalli O, Rivera-Vega L, Bhandary B, Bautista MA, Mamidala P, Michel AP, et al. Cloning and characterization of mariner-like elements in the soybean aphid, Aphis glycines Matsumura. Bull Entomol Res. 2011;101(6):697-704.

34. Kharrat I, Mezghani M, Casse N, Denis F, Caruso A, Makni H, et al. Characterization of mariner-like transposons of the mauritiana subfamily in seven tree aphid species. Genetica. 2015;143(1):63-72.

35. Richards S, Gibbs RA, Gerardo NM, Moran N, Nakabachi A, Stern D, et al. Genome sequence of the pea aphid Acyrthosiphon pisum. PLoS Biol. 2010; 8(2):e1000313.

36. Jurka J. mariner families from Acyrthosiphon pisum. Repbase Reports. 2008; 8(3):340.

37. Nicholson SJ, Nickerson ML, Dean M, Song Y, Hoyt PR, Rhee H, et al. The genome of Diuraphis noxia, a global aphid pest of small grains. BMC Genomics. 2015;16(1):1.

38. Kim H, Lee S, Jang Y. Macroevolutionary patterns in the Aphidini aphids (Hemiptera: Aphididae): Diversification, host association, and biogeographic origins. PLoS One. 2011;6(9):e24749.

39. Larsson A. AliView: A fast and lightweight alignment viewer and editor for large datasets. Bioinformatics. 2014;30(22):3276-8.

40. Edgar RC. Search and clustering orders of magnitude faster than BLAST. Bioinformatics. 2010;26(19):2460-1.
41. Wallau GL, Capy P, Loreto E, Hua-Van A. Genomic landscape and evolutionary dynamics of mariner transposable elements within the Drosophila genus. BMC Genomics. 2014;15(1):1.

42. Bannai H, Tamada Y, Maruyama O, Nakai K, Miyano S. Extensive feature detection of N-terminal protein sorting signals. Bioinformatics. 2002;18(2):298-05.

43. Gao Y, Mathee K, Narasimhan G, Wang X. Motif detection in protein sequences. In Proceedings of the Sixth Intl. Symp. on String Processing and Information Retreiaval; 1999. p. 63-72.

44. Narasimhan G, Bu C, Gao Y, Wang X, Xu N, Mathee K. Mining protein sequences for motifs. J Comput Biol. 2002;9(5):707-20.

45. Abascal F, Zardoya R, Posada D. ProtTest: Selection of best-fit models of protein evolution. Bioinformatics. 2005;21(9):2104-5.

46. Tamura K, Stecher G, Peterson D, Filipski A, Kumar S. MEGA6: Molecular evolutionary genetics analysis version 6.0. Mol Biol Evol. 2013;30(12):2725-9.

47. Robertson HM. The mariner transposable element is widespread in insects. Nature. 1993;362(6417):241-5.

48. Hartl DL, Lozovskaya ER, Nurminsky DI, Lohe AR. What restricts the activity of mariner-like transposable elements? Trends Genet. 1997;13(5):197-01.

49. Negoua A, Rouault JD, Chakir M, Capy P. Internal deletions of transposable elements: The case of Lemi elements. Genetica. 2013;141(7-9):369-79.

50. Le Rouzic A, Boutin TS, Capy P. Long-term evolution of transposable elements. Proc Natl Acad Sci U S A. 2007:104(49):19375-80.

51. Kidwell MG. Transposable elements and the evolution of genome size in eukaryotes. Genetica. 2002;115:49-63.

52. Vieira C, Nardon C, Arpin C, Lepetit D, Biémont C. Evolution of genome size in Drosophila. Is the invader's genome being invaded by transposable elements? Mol Biol Evol. 2002;19(7):1154-61.

53. Abrusán G, Krambeck HJ. Competition may determine the diversity of transposable elements. Theor Popul Biol. 2006;70(3):364-75.

54. Zhang $H H$, Shen $Y H$, Xiong XM, Han MJ, Zhang XG. Identification and evolutionary history of the DD41D transposons in insects. Genes Genomics. 2016;38(2):109-17.

55. Capy $P$, Anxolabéhère $D$, Langin $T$. The strange phylogenies of transposable elements: Are horizontal transfers the only explanation? Trends Genet. 1994;10(1):7-12.

56. Green CL, Frommer M. The genome of the Queensland fruit fly Bactrocera tryoni contains multiple representatives of the mariner family of transposable elements. Insect Mol Biol. 2001;10(4):371-86.

57. Prasad MD, Nurminsky DL, Nagaraju J. Characterization and molecular phylogenetic analysis of mariner elements from wild and domesticated species of silkmoths. Mol Phylogenet Evol. 2002;25(1):210-7.

58. Rozhkov NV, Aravin AA, Zelentsova ES, Schostak NG, Sachidanandam R, McCombie WR, et al. Small RNA-based silencing strategies for transposons in the process of invading Drosophila species. RNA. 2010;16(8):1634-45.

59. Tóth KF, Pezic D, Stuwe E, Webster A. The piRNA pathway guards the germline genome against transposable elements. In: Non-coding RNA and the Reproductive System. Springer Netherlands: 2016. p. 51-77.

60. Capy P, David JR, Hartl DL. Evolution of the transposable element mariner in the Drosophila melanogaster species group. Genetica. 1992;86:37-46.

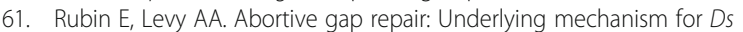
element formation. Mol Cell Biol. 1997;17(11):6294-02.

62. Brunet F, Giraud T, Godin F, Capy P. Do deletions of Mos 1-like elements occur randomly in the Drosophilidae Family? J Mol Evol. 2002;54(2):227-34.

63. Silva JC, Loreto EL, Clark JB. Factors that affect the horizontal transfer of transposable elements. Curr Issues Mol Biol. 2004;6(1):57-71.

64. Loreto ELS, Carareto CMA, Capy P. Revisiting horizontal transfer of transposable elements in Drosophila. Heredity. 2008;100(6):545-54.

65. Nicholas KB, Nicholas HBJ, Deerfield DW. GeneDoc: Analysis and visualization of genetic variation. EMBNEWNEWS. 1997:4(1):14 